MUZIKOLOŠKI ZBORNIK • MUSICOLOGICAL ANNUAL XL

UDK 94(=163.6)

Vincenc Rajšp

Slowenisches Wissenschaftsinstitut in Wien

Slovenski znanstveni inštitut na Dunaju

\title{
Die Slowenen in Mitteleuropa seit dem 13. Jahrhundert
}

\section{Slovenci v Srednji Evropi od 13. stoletja}

ZUSAMMENFASSUNG

Der Begriff Mitteleuropa kommt in der slowenischen Geschichte und vor allem im Geschichtsunterricht nur selten vor. Auch der Name jenes Staates, zu dem der überwiegende Teil des slowenischen ethnischen Gebietes gehörte, wird in den Schulbüchern meist mangelhaft und inkonsequent angegeben. Statt der Bezeichnung Heiliges Römisches Reich (Sacrum Romanum Imperium) trifft man die Namen wie Römisch-deutsches Reich, Deutscher Staat, Heiliges Römisches Reich deutscher Nation etc. Der Name des Staates ist keineswegs irrelevant, da er für das Verständnis der historischen Abläufe in diesem Raum von Bedeutung ist. Aber auch der Begriff Mitteleuropa ist nicht überflüssig, wirkten sich doch auf dem kulturellen Gebiet die Einflüsse des breiteren Raumes, den wir als Mitteleuropa bezeichnen, nachhaltig aus.

\author{
POVZETEK
}

Dieser Titel bringt mich etwas in Verlegenheit. Er enthält nämlich zwei Unbekannte, erstens die Frage, ob und auf welche Weise man im Zeitraum vom Mittelalter bis zum sogenannten nationalen Erwachen im 18. Jahrhundert und zur endgültigen Formierung der Nation im 19. Jahrhundert von "Slowenen" sprechen kann. Zum Anderen stellt auch der Begriff Mitteleuropa selbst eine Unbekannte dar, sowohl hinsichtlich seines Inhaltes wie seiner Grenze. Dazu kommt noch eine dritte Unbekannte, die aus dem Titel nicht ersichtlich ist, nämlich der Name jenes Staates, in dem die Slowenen seit dem 13. Jahrhundert lebten.

Beginnen wir mit der letzten Frage, mit der Frage des Staatsnamens, so heißen die offiziellen Bezeichnungen der Staaten bis zum Ende des Ersten Weltkrieges: vom Mittelalter bis zum Jahre 1806 Sacrum Imperium Romanum, Heiliges Römisches Reich; von 1804 bis 1867 Kaisertum Österreich und von 1867 bis zum Ende der Monarchie im Jahre 1918 Österreich-Ungarn. An dieser Stelle muß aber darauf hingewiesen 
werden, daß in diesen Staaten nicht alle Slowenen lebten, so gab es zum Beispiel in der Republik Venedig Slowenen, aber auch im Königreich Ungarn und nicht zuletzt in Frankreich während der Zeit der Illyrischen Provinzen.

In der slowenischen Historiographie werden diese Bezeichnungen eher inkonsequent verwendet. Die Fehler treten am häufigsten bei "Sacrum Romanum Imperium " (Sveto rimsko cesarstvo) auf, jenem Staat also, in dem die Slowenen am längsten lebten. Der Begriff Imperium Romanum setzte sich im Jahre 1027 durch, die zusätzliche Bezeichnung Sacrum aber, die bis zum Ende des Reiches im Jahre 1806 beibehalten wurde, seit $1254 .^{1}$ In den Geschichts- und Schulbüchern trifft man statt des offiziellen Staatsnamens diverse Ersatzbezeichnungen: Römisch-Deutsches Reich, deutscher Staat, Heiliges römisches Reich deutscher Nation etc. Am konsequentesten wird der ungenau Name auf den Karten des Historischen Schulatlas ${ }^{2}$ verwendet, nämlich Römisch-deutsches Kaiserreich. ${ }^{3}$ In der Weltgeschichte aus dem Jahre 1976 wird der Name des Staates in den meisten Fällen ungenau verwendet: Deutscher Staat am Beginn des 11. Jahrhunderts, ${ }^{4}$ die Kämpfe um den deutschen Thron im 12. Jahrhundert, ${ }^{5}$ Heiliges Römisches Reich deutscher Nation in der zweiten Hälfte des 15. Jahrhunderts $^{6}$ etc. Auch das Schulbuch für die zweite Klasse des Gymnasiums ${ }^{7}$ verwendet im Mittelalter diverse Namen für das Heilige Römische Reich: Deutsches Reich unter der Dynastie der Salier (1024-1125) ${ }^{8}$, das Reich (deutscher Nation) für die Zeit der Kreuzzüge ${ }^{9}$, Deutsches Reich (um das Jahr 1300), ${ }^{10}$ Heiliges Römisches Reich deutscher Nation ${ }^{11}$ für die Zeit des Hundertjährigen Krieges zwischen England und Frankreich, aber auch Heiliges Römisches Reich (für die Zeit der Reformation) und die Grenzen des Heiligen Römischen Reiches. ${ }^{12}$ Die richtige Bezeichnung, Das Heilige Römische Reich ist richtig angeführt für die Zeit der Reformation ${ }^{13}$ und im Kapitel "Die Entwicklung des Absolutismus in Spanien. " ${ }^{14}$ Inkonsequent ist auch die Benennung der Gebiete der habsburgischen Erblande, zu denen Steiermark, Kärnten, Krain, Görz und Triest gehörten: diese werden (richtig) als habsburgische Länder für die Zeit vom 16. - 18. Jahrhundert, ${ }^{15}$ oder aber kurz als Österreich bezeichnet, was nicht ganz richtig ist, da dieser Name nicht selbständig verwendet wurde, sondern im Zusammenhang mit

\footnotetext{
${ }^{1}$ Österreich Lexikon, Hrsg. Richard und Maria Bamberger, Ernst Bruckmüller, Karl Gutkas, Wien 1995, Heiliges Römisches Reich, Bd. I., 490.

${ }^{2}$ Ljubljana, 1994, "Die Schulbuch- und Lehrbehelfe- Kommission beim Fachbeirat der Republik Slowenien für Erziehung und Bildung befaßte sich in der Sitzung vom 8. 6. 1994 mit dem Historischen Schulatlas als didaktisches Mittel und empfahl seine Verwendung für die Schüler der Volks- und Mittelschulen.“

${ }^{3}$ Karte: Europa um das Jahr 1000, 17; Europa in der Zeit des Hundertjährigen Krieges, 18; Die Kreuzzüge (1094-1204), 19; Europa um das Jahr 1700, 29 und Frankreich in den Jahren 1789-1797, 31.

${ }^{4}$ Svetovna zgodovina [Die Weltgeschichte], Ljubljana 1976, 242

${ }^{5}$ Svetovna zgodovina 1976, 243.

${ }^{6}$ Svetovna zgodovina 1976, 308.

${ }^{7}$ Andrej Hozjan, Dragan Potočnik, Zgodovina 2

${ }^{8}$ Zgodovina 2, 12 .

${ }^{9}$ Zgodovina 2, 29.

${ }^{10}$ Zgodovina 2, 130

${ }^{11}$ Zgodovina 2, 80.

12 Zgodovina 2, 175, 212.

${ }^{13}$ Zgodovina 2, 169.

${ }^{14}$ Zgodovina 2, 206

${ }^{15}$ Zgodovina 2, 169.
} 
dem Herrscherhaus: etwa "Herrschaft zu Österreich"im 14. und 15. Jahrhundert, im 15. Jahrhundert Haus Österreich für das Herrschaftsgebiet, seit dem 16. Jahrhundert aber für alle Gebiete der Habsburger im West- und Südeuropa (auch in anderen Sprachen, etwa: Casa de Austria, Casa d'Austria, Maison d'Autriche). Niemals bezog sich aber der Name auf das Gebiet des heutigen Österreich. Die inkonsequente Verwendung der Namen verursacht bei den Schülern zweifelsohne zusätzliche Probleme.

Die Bezeichnung Heiliges Römisches Reich wird im Großen historischen Weltatlas größtenteils richtig verwendet. ${ }^{16}$ Aber auch hier findet sich auf den Karten "Der Aufstieg des christlichen Westens vom 11. bis 13. Jahrhundert “, ${ }^{17}$ oder "Frankreich im Jahre 1180 " ${ }^{18}$ der Name Deutsches Königreich. Ebenso trägt die Karte des Heiligen Römischen Reiches im Jahre 1648, die auch die slowenischen Länder umfaßt, den Titel Deutschland im Jahre $1648^{19}$.

Der Begriff Mitteleuropa läßt sich für den Historiker ziemlich schwer fassen, da er sich politischen oder geographischen Abgrenzungen entzieht. Er ist vielmehr als Kultur- oder kulturgeographischer bzw. kulturhistorischer Begriff tauglich. Der Begriff Mitteleuropa kommt im Historischen Schulatlas, im Schulbuch für Geschichte 2 oder im Großen Atlas der Weltgeschichte nicht vor. Die Enzyklopädie Sloweniens behandelt diesen Begriff erst seit dem Ende des 18. Jahrhundert ${ }^{20}$ als "den mittleren Teil Europas zwischen dem Mittelmeer und dem Baltikum«. Für die Geschichte der Slowenen ist er wenig aussagekräftig, abgesehen von ihrer zweifelsfreien Zugehörigkeit zu diesem Raum. Keineswegs ist aber Mitteleuropa als Begriff leer und überflüssig. Im Historischen Atlas (Putzger) ${ }^{21}$ kommt er häufig in der Überschrift der Karten vor, so zum Beispiel: Mittel- und Westeuropa vom 11.-13. Jahrhundert; ${ }^{22}$ hier werden als Mitteleuropa das Heilige Römische Reich, aber auch Teile Polens, Ungarns und Serbiens bezeichnet; Mitteleuropa um das Jahr $1400^{23}$ zählt zum Heiligen Römischen Reich, Polen und Ungarn auch einen Teil Bosniens dazu; Mitteleuropa im 16. Jahrhundert (Wirtschaft), ${ }^{24}$ Mitteleuropa am Beginn der Französischen Revolution 1789, ${ }^{25}$ Mitteleuropa nach dem 30-jährigen Krieg $1648^{26}$, wobei die Grenzen dieses Mitteleuropa nirgends klar verzeichnet sind. Auf den Karten dargestellt sind aber auch Teile Polens und Ungarns, obwohl der Großteil der Texte das Gebiet des Heiligen Römischen Reiches betrifft. In der slowenischen Ausgabe des Großen Atlas zur Weltgeschichte wurde der Begriff "Mitteleuropa" bei den Karten nicht gefunden.

\footnotetext{
${ }^{16}$ Veliki Atlas svetovne zgodovine [Der große historische Weltatlas], Ljubljana 1999.

${ }^{17}$ Veliki Atlas svetovne zgodovine, 72.

${ }^{18}$ Veliki Atlas svetovne zgodovine, 84 .

${ }^{19}$ Veliki Atlas svetovne zgodovine, 113.

${ }^{20}$ Enciklopedija Slovenije 12, Ljubljana 1998, "Srednja Evropa".

${ }^{21}$ Putzger, Historischer Weltatlas Berlin, 103. Ausgabe, 2001.

${ }_{22}^{22}$ Mittel- und Westeuropa 11. - 13. Jahrhundert, Putzger, 62.

${ }^{23}$ Mitteleuropa um 1400, Putzger, 80.

${ }^{24}$ Mitteleuropa im 16. Jahrhundert (um 1550) /Wirtschaft/, Putzger, 92.

${ }^{25}$ Mitteleuropa bei Beginn der Französischen Revolution 1789, Putzger, 120.

${ }^{26}$ Mitteleuropa nach dem 30-jährigen Krieg 1648, Putzger, 98.
} 
MUZIKOLOŠKI ZBORNIK • MUSICOLOGICAL ANNUAL XL

In der Zeit der europäischen Vereinigung bekommt der Begriff "Mitteleuropa" neue Dimensionen. Die bestehenden politischen Grenzen teilen häufig Kulturräume, die eine lange gemeinsame Geschichte und Kultur hatten. Deshalb sind heute Bestrebungen im Gange, daß Europa neben einer geographischen Karte der europäischen Staaten auch eine Karte der europäischen Kulturräume bekommt.

Für eine dieser Karten ist der Titel Kulturraum Mitteleuropa vorgesehen. Um diesen Raum abzugrenzen, wurden zehn Kriterien erstellt. Demnach handelt es sich um den geographischen und politischen Raum, der sich zwischen den Kolonialmächten im Westen und Rußland sowie dem Osmanischen Reich im Osten befindet und als Gebiet ohne Überseeorientierung bezeichnet wird, etc. Nach diesen Kriterien gehören dazu Teile von

Italien: Südtirol, Trentino, Gebiet von Vicenza, Belluno, Friaul und Triest,

Bosnien und Hercegowina: vor allem die kroatischen Gebiete der Hercegowina,

Serbien und Mentenegro: Vojvodina,

Rumänien: Gebiete im Karpatenbogen

Rumänien, Ukraine: Bukowina,

Ukraine: Galizien und Karpato-Ukraine

Weißrußland: Gebiet um Grodno

Niederlande: Groningen und Friesland, sowie das Gebiet um Maastricht

Belgien: deutschsprachige Gebiete mit deutscher Amtssprache,

Frankreich: Ostlothringen und Elsass. ${ }^{27}$

Für die Slowenen besonders interessant ist die Tatsache, daß die Republik Venedig nicht zum mitteleuropäischen Raum gezählt wird; nicht einmal in diesem weiten Rahmen sind alle Slowenen vereinigt.

Noch einige Gedanken zum Begriff "Slowenen." Auch diese Bezeichnung ist nicht eindeutig - seit dem fernen 13. Jahrhundert bis zum heutigen Tag. Wann der Begriff Slowenen (Slouenci) auftauchte, den Primož Trubar zum ersten Mal im Jahre 1550 verwendete und deren Sprache er im Jahre 1555 als slowenische Sprache (slouenski jezig) ${ }^{28}$ bezeichnete, ist nicht geklärt. Wo die Grenzen dieser Sprachgruppe verliefen, überlieferte auch Trubar nicht. Jedenfalls handelt es sich hier nicht um einen Nationsbegriff für die Slowenen. Der Begriff "Nation", der sich durch eine gemeinsame Sprache, ein gemeinsames Siedlungsgebiet, ein gemeinsames historisches Gedächtnis, bestimmte Formen der kulturellen und wirtschaftlichen Verflechtung sowie durch ein Gemeinschafts- oder Nationalbewußtsein und den Willen zum Zusammenleben manifestiert, bildete sich in Europa im 18. und 19. Jahrhundert aus. ${ }^{29}$

Deshalb kann im Mittelalter weder von einer slowenischen noch von irgend einer anderen, etwa der deutschen Nation, die Rede sein. Es ist auch sinnlos, von einem deutschen Staat oder einem Staat deutscher Nation u. ä zu sprechen. Mit dem Begriff natio wurde damals die Landeszugehörigkeit bezeichnet, was die Daten über die

\footnotetext{
${ }^{27}$ Peter Jordan, Kulturäumliche Großgliederung Europas. Unveröffentlichtes Manuskript.

${ }^{28}$ Enciklopedija Slovenije 11, Ljubljana 1997, 165.

${ }^{29}$ Enciklopedija Slovenije 7, Ljubljana 295.
} 
inskribierten Studenten auf den Universitäten oder seit dem 16. Jahrhundert in den Katalogen der Jesuiten bezeugen.

Zweifellos kann aber mit dem Begriff Slowenen schon seit dem Mittelalter die Bevölkerung des slowenischen Sprachgebietes, die Slowenisch, in manchen Gebieten aber auch Deutsch, Italienisch, Friaulisch oder im Osten Ungarisch sprach, bezeichnet werden.

Nach der Enzyklopädie Sloweniens bildete sich die "slowenische Identität in jenen kulturellen, politischen und wirtschaftlichen Verhältnissen aus, die seit dem Mittelalter die Zivilisation des europäischen Westens im Übergangsraum zu ihren östlichen und Grenzgebieten kennzeichneten." ${ }^{30}$ Wobei aber natürlich berücksichtigt werden muß, daß sich in diesem Sinne die Identität aller Gesellschaftsschichten in diesem Raum ausgebildet hat.

Trotz der Geschlossenheit des Sprachgebietes waren die Slowenen schon seit dem Mittelalter auf verschiedene politische Einheiten aufgeteilt und zwar auf einzelne Länder des so genannten Innerösterreich: die Steiermark, Kärnten, Krain, das Land Görz und Triest, die seit dem 16. Jahrhundert unter der Herrschaft der Habsburger als Teil der Erbländer wohl vereint war. Aber schon im Mittelalter war die Aufteilung der Slowenen auf verschiedene Staaten zu beobachten: der Großteil der Slowenen lebte im Heiligen Römischen Reich, ein kleiner Teil im Übermurgebiet kam unter das Königreich Ungarn, während die westlichen von Slowenen besiedelten Gebiete nach dem Untergang des Patriarchates von Aquileia als weltliche Macht im Staat der Republik Venedig lagen.

Daher vermischten sich bei den Slowenen, die sich im 19. Jahrhundert zu einer reifen europäischen Nation entwickelten, in der Vergangenheit verschiedene Formen der europäischen Kultur. Die Folge dieser Aufteilung, aber auch der feudalen Zersplitterung, waren breite Kontaktzonen. Zum Einen übernahm der slowenische Raum die europäische Kultur, doch parallel dazu wirkten zahlreiche, auf dem slowenischen Siedlungsgebiet geborene (vor allem Männer) im europäischen oder zumindest mitteleuropäischen Raum.

Im Mittelalter holte das slowenische Gebiet in wirtschaftlicher und kulturellen Hinsicht den übrigen europäischen Raum ein. Es entstanden Klöster als Zentren der wirtschaftlichen und geistigen Kultur. Die günstige Verkehrslage ermöglichte die enge Verflechtung des slowenischen Raumes mit den Nachbarländern. Schon seit dem Mittelalter verliefen bedeutende Handelswege über das slowenische Gebiet. Der slowenische Bauer konnte als Säumer sowohl die Nachbarregionen als auch die "fernen" Länder kennenlernen. Die Bauern von Bischoflack kamen so bis Freising, für die Krainer Säumer waren aber auch die venezianischen Städte interessant. Ihre große Erfindungsgabe und Unternehmungslust werden in dicken Faszikeln über das Konterbandenwesen dokumentiert. Im 18. Jahrhundert wurden manche Wege der Schmuggler sogar kartographisch verewigt.

\footnotetext{
${ }^{30}$ Enciklopedija Slovenije 11, Ljubljana 1997, 166.
} 
Die Mobilität der slowenischen Bevölkerung seit dem Mittelalter bezeugen auch die zahlreichen Pilgerwege: regelmäßige Wallfahrt (alle sieben Jahre) nach Köln (Kelmorain), zum Hl. Wolfgang (Bolfenk) nach Bayern, nach Rom, und aus den Städten des Küstenlandes sogar nach Jerusalem. Wie die Aufzeichnungen aus der Reformationszeit zeigen, war auch damals das Verlangen nach Wallfahrten groß und für die Reformatoren ein Beweis des Aberglaubens. In der darauffolgenden Epoche bis zu den Kirchenreformen des Josef II. war das Pilgern ganz besonders beliebt. Jene Grenze, die als kommende Schengengrenze im Südosten und Süden Sloweniens erwartet wird, war (außer in Istrien) die fast tausendjährige Grenze des Heiligen Römischen Reiches. Aber auch diese Grenze schloß das Staatsgebiet nicht ab, eine lebhafte Kommunikation der Bevölkerung auf beiden Seiten war durchaus üblich. Seit der Mitte des 15. Jahrhunderts war diese Grenze fast ein Jahrhundert so durchlässig, daß sie der Bevölkerung diesseits der Grenze keinen Schutz vor feindlichen Einfällen aus dem Süden bot.

Der slowenische Raum war aber nicht nur durch Ereignisse mit ungünstigen Folgen mit dem Süden verflochten. Es wurden auch geistige Kontakte geknüpft, die Jahrhunderte anhielten. So stellten z. B. die Franziskaner, die aus Bosnien in die slowenischen Gebiete geflüchtet waren, enge Verbindungen her. Kulturelle Kontakte mit den südslawischen Nachbarn waren auch in Istrien sehr stark, wo die Verfechter der glagolitischen Schrift aus Dalmatien unter der slowenischen Bevölkerung tätig waren.

Auch das Übermurgebiet, das im Königreich Ungarn lag, stand in enger Verbindung mit dem südlichen Nachbarn. Der überwiegende Teil des Übermurgebietes gehörte bis zur zweiten Hälfte des 18. Jahrhunderts zur Diözese Zagreb/Agram. Bedeutsam waren auch andere kulturelle Berührungen, wie zum Beispiel die Bücher des in Ormož geborenen und in Varaždin verstorbenen Anton Vramec (1538-1587) in der kaj-kroatischen (kajkavischen) Sprache (er selbst bezeichnete die Sprache als Slowenisch - Szlouenzki jezik).

Das slowenische Gebiet war eng in die kulturellen und geistigen Bewegungen des 15. und 16. Jahrhundert eingebunden. Der Humanismus setzte sich nicht nur auf dem Gebiet der Republik Venedig und in Triest durch, sondern auch im Landesinneren (in der Diözese Laibach/Ljubljana durch die Beschöfe Lamberg, Raubar etc.). Zahlreiche bedeutende Humanisten aus den slowenischen Gebieten wirkten auf der Universität in Wien: der Propst und Kanzler der Wiener Universität Thomas Prelogar war daneben auch Erzieher des Kaiser Maximilian I., oder der Wiener Humanist Bernard Perger aus Zgornja Ščavnica, der u.a. Rektor gewesen war, und andere.

Den stärksten Widerhall im 16. Jahrhundert fand im slowenischen Raum die Reformation. Die ersten slowenischen Bücher wurden gedruckt. Der überaus aktive Schreiber und Organisator Primož Trubar vermittelte in seinen Briefen an angesehene Persönlichkeiten, Fürsten und sogar den Kaiser Informationen über die Verhältnisse im Land. In den Einführungen zu den Büchern wandte er sich mit seinen Schilderungen an die breitere Bevölkerung im Staat. Die Kontakte waren aber nicht nur in die nördliche sondern ebenso in die südliche Richtung ausgerichtet, ein bedeutendes Opus bilden Bücher in kyrillischer und glagolitischer Schrift. 
MUZIKOLOŠKI ZBORNIK • MUSICOLOGICAL ANNUAL XL

In der Zeit der Gegenreformation spielte das geschriebene Wort nicht die zentrale Rolle im kirchlichen und geistigen Leben. Größere Bedeutung bekamen die neuen Barockkirchen, die mit Malereien ausgeschmückt wurden.

Mit den josephinischen Reformen gewann das geschriebene Wort in der Volkssprache erneut an Bedeutung. Man kann wohl mit Recht feststellen, daß sowohl die Initiative des Laibacher Bischofs Karl von Herberstein, die kirchlichen Bücher und die Bibel ins Slowenische zu übersetzen, wie die Vorschläge des Klagenfurter Bischofs Auersperg zur Reorganisation der Diözesen (auch unter Berücksichtigung der Sprachverhältnisse), oder die slowenischen Ortsbezeichnungen auf den Karten von Haqué für Krain nach dem Jahre 1770, als auch die Aufzeichnung der slowenischen Namen auf den Militärkarten zur Zeit des Joseph II., die Epoche eines beschleunigten Wachstums und der Entwicklung der Slowenen zu einer entwickelten Nation am Ende des 19. Jahrhundert einleiteten.

Trotz diverser ungünstiger Gegebenheiten in der Geschichte, unter anderem das Fehlen eines slowenischen wirtschaftlichen und kulturellen Zentrums, aber auch eines kirchliches Zentrums innerhalb des Sprachgebietes, trotz Aufsplitterung und des Mangels einer slowenischer Oberschicht, erlangten die Slowenen im Laufe einer nicht geradlinig verlaufenden Entwicklung ihren Nationalstaat.

Heute stehen die Slowenen vor neuen Herausforderungen der politischen Realität, eines Lebens im Vereinigten Europa. Ein Bestandteil der Nationsbildung war auch die nationale Grenze. Die Slowenen bekamen die bestehenden Grenzen erst nach dem Zweiten Weltkrieg, die in nationaler Hinsicht keineswegs optimal verliefen. Aber auch diese Grenzen verfestigten sich. Nach dem Eintritt in die Europäische Union verlieren sie aber ihre Bedeutung, was bei Manchen Unbehagen auslöst.

Wenn der Titel Die Slowenen im Mitteleuropa lautete, so befinden sich die Slowenen eigentlich dort, wo sie schon einmal waren. Mit den Nachbarn konnte auch in ihren Sprachen kommuniziert werden, in mancherlei Hinsicht beeinflußten auch die Kulturräume des Nachbarn den slowenischen Raum und umgekehrt. Das Bewußtsein dieser Vergangenheit und die Erfahrungen lassen die Bevölkerung des slowenischen Raumes mit Optimismus in die Zukunft blicken. Er soll dort, wo es keine Grenzen mehr geben wird, das ängstliche Abschotten hinter der eigenen Grenze in die optimistische Kreativität einer nach außen hin orientierten Zusammenarbeit verwandeln. Wo die Grenzen weiter bestehen werden, sollte ein möglichst kreatives Miteinander über diese Grenzen hinweg möglich sein. Der Begriff "Die Slowenen in Mitteleuropa" erlaubt gewiß keine "vernagelte" Einstellung. 